\title{
Simultaneous Mapping and Redirected Walking for ad hoc Free Walking in Virtual Environments
}

\section{Conference Paper}

Author(s):

Nescher, Thomas; Zank, Markus; Kunz, Andreas (i)

Publication date:

2016

Permanent link:

https://doi.org/10.3929/ethz-a-010616183

Rights / license:

In Copyright - Non-Commercial Use Permitted 


\title{
Simultaneous Mapping and Redirected Walking for ad hoc Free Walking in Virtual Environments
}

\author{
Thomas Nescher* $\quad$ Markus Zank $^{\dagger} \quad$ Andreas Kunz
}

ICVR - IWF - ETH Zurich

\begin{abstract}
Providing real walking in virtual environments remains a challenge because of space, setup, and tracking system requirements. With the help of redirected walking (RDW), natural and unconstrained walking in virtual environments has become possible - without using mechanical locomotion devices - by manipulating the user's real world trajectory such that he remains within the boundaries of the walkable space. Nevertheless, typical home or office environments do not provide sufficient space or a suitable room which is free of obstacles.

This paper presents an approach that combines RDW with a low-cost and user-worn tracking approach based on simultaneous localization and mapping (SLAM). I.e. learning the environment with the walkable area, tracking the user's viewpoint, and RDW is done on the fly without any prior setup, without preparing a room, and without setting up a tracking system. This allows ad hoc free walking in virtual environments even within dynamic and cluttered physical rooms, where the walkable area is of arbitrary shape.

Furthermore, by combining SLAM and a planning RDW controller, this approach has the potential to provide the best free walking experience for any given physical environment.
\end{abstract}

Keywords: SLAM, redirected walking, tracking, head tracking, virtual reality, locomotion, walkable area, obstacle avoidance.

Index Terms: H.5.1 [Information Interfaces and Presentation]: Multimedia Information Systems-Artificial, augmented, and virtual realities; I.3.7 [Computer Graphics]: Three-Dimensional Graphics and Realism-Virtual reality; I.4.8 [Image Processing and Computer Vision]: Scene Analysis-Tracking

\section{INTRODUCTION}

Enabling a user to really walk inside an immersive virtual environment provides a fascinating user experience and was shown to have several advantages over alternative navigation metaphors. For instance, it allows easier navigation and is more presence-enhancing [6]. Typically, such virtual reality (VR) systems are realized by tracking a user's viewpoint, rendering a scene from the tracked viewpoint in real time, and presenting it to the user on a head mounted display (HMD). To avoid using mechanical locomotion devices but still allow a user to freely explore virtual environments (VEs) that are larger than the tracked space, Razzaque et al. [5] proposed redirected walking (RDW). RDW employs so-called redirection techniques, see [7], to keep a user within the boundaries of the walkable area or the physical room.

Recent developments in consumer hardware like HMDs, depth cameras, and mobile devices have greatly reduced the cost of

\footnotetext{
*e-mail: nescher@iwf.mavt.ethz.ch

†e-mail: zank@iwf.mavt.ethz.ch

‡e-mail: kunz@iwf.mavt.ethz.ch
}

building a VR system. Nevertheless, it remains costly and timeconsuming to set up a suitable tracking system and prepare a tracked space which serves as a walkable area. The walkable area should have a decent size to allow for free exploration of VEs and must be free of obstacles. Furthermore, its dimensions must be determined in advance so that RDW controllers are aware of the boundaries and can steer the user accordingly. In a typical home or office environment these requirements are not fulfilled. These environments are dynamic. Tables, chairs, or other equipment is moved and there is often not enough space or money available to maintain a dedicated tracking space free of obstacles.

For providing the best immersive experience, VR systems have high requirements on the performance of the tracking system. Hence, usually complex and permanently mounted tracking systems are used. In order to reduce costs and setup times of such systems, wearable and self-contained tracking approaches were proposed, For instance, some use inertial sensors attached to user's feet $[1,3]$ or a user-worn position tracker [8]. As these techniques track the user's position relative to some initial position, they still require some additional tracking or an external reference to correct drift and relate the relative position to an absolute room position. The layout of the walkable space must be known in advance as well.

A different approach, widely used in robotics, provides an alternative. So-called simultaneous localization and mapping (SLAM) methods build and update a map of an unknown environment while at the same time keeping track of the tracking device's location within the environment (the learned map). SLAM methods can work with various sensors but often optical sensors like cameras, depth cameras or laser range scanners are used. Therefore, SLAM methods combined with mobile hardware, for sensing and mapping the 3D structure of an environment, allow for self-contained tracking of a user's viewpoint.

This paper presents an approach that combines SLAM with RDW, including mapping the walkable area, for free walking in VEs within arbitrary physical environments. Hence, it is no longer necessary to set up a tracking system or prepare a room in advance.

\section{VR SYSTEM}

The VR system is composed of an HMD, a backpack with a notebook for rendering the scene, and Google's project Tango ${ }^{1}$ tablet computer (model "Yellowstone"). The Tango tablet is attached to the HMD as shown in Figure 1(a) so that its sensing hardware faces forward. The tablet features a color camera and a motion and depth sensing camera. It includes software for SLAM which builds and/or updates a 3D map of the environment. The resulting map is essentially a mesh which can be stored for later use (i.e. later tracking in a known environment) or shared with other users. The tablet sends its position and orientation estimates using WLAN to the notebook in real time. Figure 2 (left) shows the view of the Tango tablet along a corridor in an apartment. The black areas indicate yet unmapped areas because they are out of range of the depth sensing camera. The range of the depth camera is only a few meters. Figure 2 (right) shows a top-down view of the generated 3D map after the user walked along the corridor into a room.

${ }^{1}$ Google's Project Tango: https://www.google.com/atap/project-tango/ 


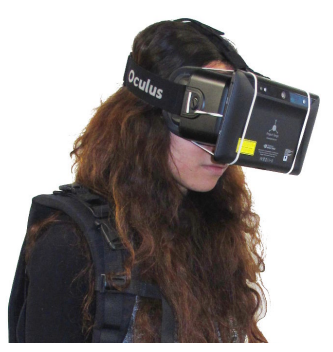

(a) VR system mock-up.

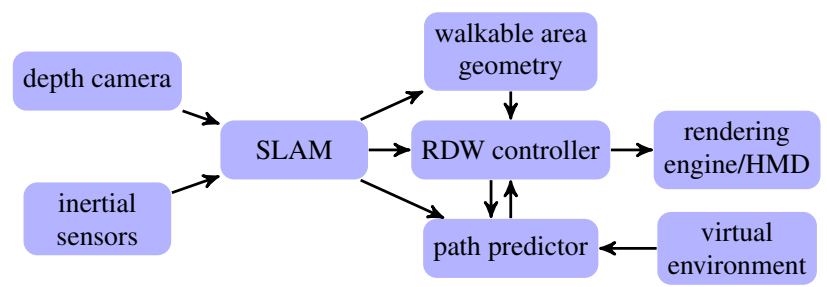

(b) System overview.

Figure 1: Wearable VR system mock-up, composed of an HMD with the Tango tablet device attached to it and overview of the software.

In order to determine the walkable area, which is free of obstacles like walls or chairs, a walking plane (normal to gravity vector) is fitted to the lowest plane in the initial mesh. This plane essentially normalizes the coordinate system so that the floor is at height 0 . The walkable area is then determined by the whole area in the mesh that has nothing above it, with exception of a small error margin due to sensor noise. However, objects that are above the user's head, like the ceiling or lamps, do not reduce the walkable area. The walkable area is continuously updated in the same fashion as the Tango tablet updates the map of the environment. I.e unmapped areas are getting mapped as well as changes in the already mapped environment. In contrast to traditional systems for real walking in VEs, which consider only walkable area versus boundaries, such a setup has to consider the types: walkable area, blocked area, and also unmapped area.

Because the environment is not known in advance, non-planning RDW controllers that steer a user towards a predefined position in the real environment are not applicable. Furthermore, they could not handle unmapped areas. A suitable RDW controller has to dynamically optimize for the best free walking experience given the currently known walkable area of arbitrary shape. For instance, MPCRed as proposed in [4] is applicable. Next, the unmapped areas have to be handled. If a boundary of the walkable area is wide enough to walk through and connects to unmapped area, there might be potentially more walkable area there. Therefore, a RDW controller should steer a user towards unmapped area from time to time to increase the known walkable area. As the area is unknown only heuristics help to guess if there might be more walkable area. One simple heuristic is to make MPCRed assume that the unmapped area connects to a $4 \mathrm{~m}$ deep room, where its width equals the width of the boundary to the unmapped area. Hence, at some point the controller will try to use that space. Because MPCRed continuously re-plans ahead for a certain time horizon, it can deal with unexpected changes. Therefore, even if the real area turns out to be smaller, it will avoid a collision of the user. However, a reset technique (see [7]) might have to be applied. Such a setup could also require using repositioning resets (see [7]) in case a user ends up in a narrow dead-end corridor. The working principle of the system is summarized in Figure 1(b).
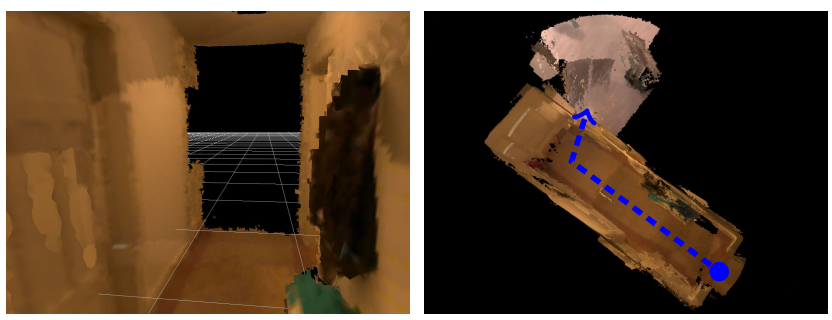

Figure 2: 3D model generated by the Tango device during a walkthrough. (left) Scene view at the beginning of a corridor. (right) Topdown view of the generated 3D model showing the corridor and parts of a room. The linearized user path is indicated by a dashed line.

\section{CONCLUSION AND FUtURE WORK}

By combining SLAM and RDW, this paper presented an approach for a self-contained and low-cost VR system that could be used without any prior setup, calibration, or maintaining a tracking space. Such a system might be especially useful for home or office environments to quickly experience a VE by walking.

The limited range, field of view, resolution, and update rate of the Tango sensors as well as the latency still limit the quality and applicability of this approach. Furthermore, the whole data processing is computationally intensive, hence using an additional userworn notebook as in the current setup is recommended. While cluttered and/or narrow environments provide many optical features and simplify the use of SLAM methods, they reduce the free walking experience as the resulting resets would disturb the user experience. In such an environment it could make sense to use alternative metaphors for navigation. For instance, navigation metaphors and visualizations of boundaries as proposed in [2]. Generally, such a system calls for new metaphors where the walkable area gets somehow visualized in the VE, e.g., by a set of paths or areas indicated with soft boundaries in the VE. These boundaries of the walkable area or the available paths could only be shown when the user wants it or when RDW is not applicable efficiently. Metaphors, e.g. in [2], could then be used to move or rotate these paths/areas in the VE.

\section{REFERENCES}

[1] E. Bachmann, M. Zmuda, J. Calusdian, X. Yun, E. Hodgson, and D. Waller. Going anywhere anywhere: Creating a low cost portable immersive ve system. In 17th International Conference on Computer Games, pages 108-115, 2012.

[2] G. Cirio, P. Vangorp, E. Chapoulie, M. Marchal, A. Lecuyer, and G. Drettakis. Walking in a cube: Novel metaphors for safely navigating large virtual environments in restricted real workspaces. IEEE Transactions on Visualization and Computer Graphics, 18(4):546-554, April 2012.

[3] E. Hodgson, E. Bachmann, D. Vincent, M. Zmuda, D. Waller, and J. Calusdian. WeaVR: a self-contained and wearable immersive virtual environment simulation system. Behavior Research Methods, pages $1-12,2014$

[4] T. Nescher, Y.-Y. Huang, and A. Kunz. Planning redirection techniques for optimal free walking experience using model predictive control. In Symposium on 3D User Interfaces, pages 111-118. IEEE, March 2014

[5] S. Razzaque, Z. Kohn, and M. C. Whitton. Redirected walking. In Proceedings of Eurographics, pages 289-294, 2001.

[6] R. A. Ruddle and S. Lessels. The benefits of using a walking interface to navigate virtual environments. ACM Transactions on Computer-Human Interaction, 16(1):1-18, 2009.

[7] E. Suma, G. Bruder, F. Steinicke, D. Krum, and M. Bolas. A taxonomy for deploying redirection techniques in immersive virtual environments. In Virtual Reality Short Papers and Posters, pages 43-46. IEEE, 2012.

[8] M. Zank, T. Nescher, and A. Kunz. Tracking human locomotion by relative positional feet tracking. In IEEE Virtual Reality, pages $317-$ 318. IEEE, March 2015. 